\title{
Immediate effects of acupuncture on biceps brachii muscle function in healthy and post- stroke subjects
}

Ana Paula S Fragoso ${ }^{1}$ and Arthur S Ferreira, ${ }^{1,2^{*}}$

\begin{abstract}
Background: The effects of acupuncture on muscle function in healthy subjects are contradictory and cannot be extrapolated to post-stroke patients. This study evaluated the immediate effects of manual acupuncture on myoelectric activity and isometric force in healthy and post-stroke patients.

Methods: A randomized clinical trial, with parallel groups, single-blinded study design, was conducted with 32 healthy subjects and 15 post-stroke patients with chronic hemiparesis. Surface electromyography from biceps brachii during maximal isometric voluntary tests was performed before and after 20-min intermittent, and manual stimulation of acupoints Quchi (LI11) or Tianquan (PC2). Pattern differentiation was performed by an automated method based on logistic regression equations.
\end{abstract}

Results: Healthy subjects showed a decrease in the root mean-squared (RMS) values after the stimulation of LI11 (pre: $1.392 \pm 0.826 \mathrm{~V} ;$ post: $0.612 \pm 0.0 .320 \mathrm{~V} ; P=0.002$ ) and PC2 (pre: $1.494 \pm 0.826 \mathrm{~V}$; post: $0.623 \pm 0.320 \mathrm{~V} ; P=0.001$ ). Elbow flexion maximal isometric voluntary contraction (MIVC) was not significantly different after acupuncture stimulation of LI11 (pre: $22.2 \pm 10.7$ kg; post: $21.7 \pm 9.5 \mathrm{~kg} ; P=0.288$ ) or PC2 (pre: $18.8 \pm 4.6$ kg; post: $18.7 \pm 6.0$ kg; $P$ $=0.468$ ). Post-stroke patients did not exhibit any significant decrease in the RMS values after the stimulation of LI11 (pre: $0.627 \pm 0.335 \mathrm{~V} ;$ post: $0.530 \pm 0.272 \mathrm{~V} ; P=0.187$ ) and PC2 (pre: $0.601 \pm 0.258 \mathrm{~V} ;$ post: $0.591 \pm 0.326 \mathrm{~V} ; P=0.398$ ). Also, no significant decrease in the MIVC value was observed after the stimulation of LI11 (pre: $9.6 \pm 3.9$ kg; post: 9.6 $\pm 4.7 \mathrm{~kg} ; P=0.499$ ) or $P C 2$ (pre: $10.7 \pm 5.6 \mathrm{~kg}$; post: $10.2 \pm 5.3 \mathrm{~kg} ; P=0.251$ ). Different frequency of patterns was observed among healthy subjects and post-stroke patients groups $\left(\chi^{2}=9.759 ; P=0.021\right)$.

Conclusion: Manual acupuncture provides sufficient neuromuscular stimuli to promote immediate changes in motor unit gross recruitment without repercussion in maximal force output in healthy subjects. Post-stroke patients did not exhibit significant reduction on the myoelectric activity and maximal force output after manual acupuncture and needs further evaluation with a larger sample.

Trial registration: Brazilian Clinical Trials Registry RBR-5g7xqh.

\section{Background}

Deaths related to cardiovascular disease are expected to be 24 million in 2030 [1,2]. The importance of management of risk factors for stroke is getting reinforcement. The rehabilitation techniques are aimed to improve by minimizing both recovery time and neurologic

\footnotetext{
* Correspondence: arthur_sf@ig.com.br

'Laboratory of Human Motion Analysis, Post-graduation Program of Rehabilitation Science, Centro Universitário Augusto Motta, Rio de Janeiro, Brazil

Full list of author information is available at the end of the article
}

impairments and maximizing functional independence of post-stroke patients $[3,4]$.

The efficacy of Chinese medicine $(\mathrm{CM})$ intervention in stroke-related functional impairments was evaluated. In the last two decades, most acupuncture controlled trials in post-stroke patients failed to obtain significant, longterm improvement of the functional aspect [5], suggesting that new methodological approaches were required, such as observation of neuromuscular activity [6-9]. Surface electromyography (sEMG) can evaluate muscle activity in health and morbid conditions in a
C Biomed Central 
noninvasive manner, allowing the assessment of the proportion and duration of muscular activity as well as the neural recruitment strategies. The root mean-squared (RMS) value of sEMG signals has been used as a timedomain parameter for monitoring changes in myoelectric activity since it reflects the relationship between muscle force and corresponding gross motor unit (MU) recruitment [10].

The immediate effects of acupuncture on the sEMG parameters were contradictory and not clearly understood in healthy subjects. Toma et al. [11] analyzed the sEMG signals of the flexor digitorum superficialis, flexor digitorum profundus, and semitendinous ( $\mathrm{n}=17$; 20-38 years old). Interventions comprised of perpendicular needle insertion into these muscles during 15 min without specified acupoints. A significant increase in the sEMG responses was observed during maximal knee flexion but no significant difference was observed for the handgrip sEMG values. Tough [12] analyzed the sEMG activity of the common wrist extensor muscles (n $=35 ; 18-70$ years old). All subjects arbitrarily received: (a) 20-min stimulation of Deqi true at acupoints Hegu (LI4) and Shousanli (LI10); (b) Deqi false or inappropriate in the acupoints Quze (PC3) and Neiguan (PC6); and (c) no stimulation (considered as a control). The results showed that sEMG was reliable (intra-class correlation coefficient $=0.9996$ ), but no significant difference was observed among the protocols. Costa and Araújo [13] investigated the sEMG signals from the tibialis anterior muscle ( $\mathrm{n}=15$ per group, $18-25$ years old). The effects of manual stimulation at acupoints Zusanli (ST36) and Yinlingquan (SP9) were evaluated by the RMS values and maximal isometric voluntary contraction (MIVC) force estimated from sEMG and force signal, respectively. A significant reduction in the RMS values was observed in both ST36 and SP9 immediately after acupuncture, but the MIVC value was significantly reduced only after the stimulation at ST36. Therefore, as related to the sEMG signals derived from those muscles, the signal should increase in tonification and decrease in sedation. However, no convincing data could support this statement and no model was proposed to explain the observed results.

The immediate effects of acupuncture on the sEMG amplitude and MIVC values in post-stroke patients have not been investigated. Also, the results from healthy subjects cannot be extrapolated to post-stroke patients due to several reasons. According to CM theory, healthy subjects may present different frequency distributions of patterns and different responses to acupuncture, compared to post-stroke patients. From the biopsychosocial model, the structural lesion observed in post-stroke patients imposes a limited control of muscle activation leading to different strategies for MU recruitment. Thus, this study was justified by the severity of motor impairments and functional disability arising from stroke.

This study aims to evaluate the immediate effects of manual stimulation of acupoints on both electrical activity and strength of the biceps brachii muscle in two parallel groups (healthy subjects and post-stroke patients with chronic hypertonic hemiparesis); and to propose a model to explain the relationship between acupoint stimulation and variables of muscle function, i.e. the RMS and MIVC values.

\section{Methods}

A detailed description of this randomized clinical trial, with two parallel groups (described as 'clinical study 1' and 'clinical study 2', respectively), single-blinded study design has been published [14]. The flowchart of this study design was depicted in Figure 1 . This study protocol followed recommendations of both the Consolidated Standards of Reporting Trials [15], Standards for Reporting Interventions in Clinical Trials of Acupuncture [16], and Declaration of Helsinki. Also, the protocol was approved by the Institutional Committee of Ethics in research before execution (CAAE-0006.0.307.000-10). All subjects read and signed the written consent form after the explanation on the research aims and methods.

\section{Casuistic}

Demographic characteristics were presented in Table 1. Q1 In clinical study $1(\mathrm{n}=40)$, healthy subjects were recruited from the academic institutional community. No pain, trauma or musculoskeletal injuries in the upper limbs, pregnancy, or any other contra-indication to acupuncture was found on them through the clinical screening. In clinical study $2(n=22)$, post-stroke patients were recruited from the Fluminense Rehabilitation Association. The patients with upper neurons lesion

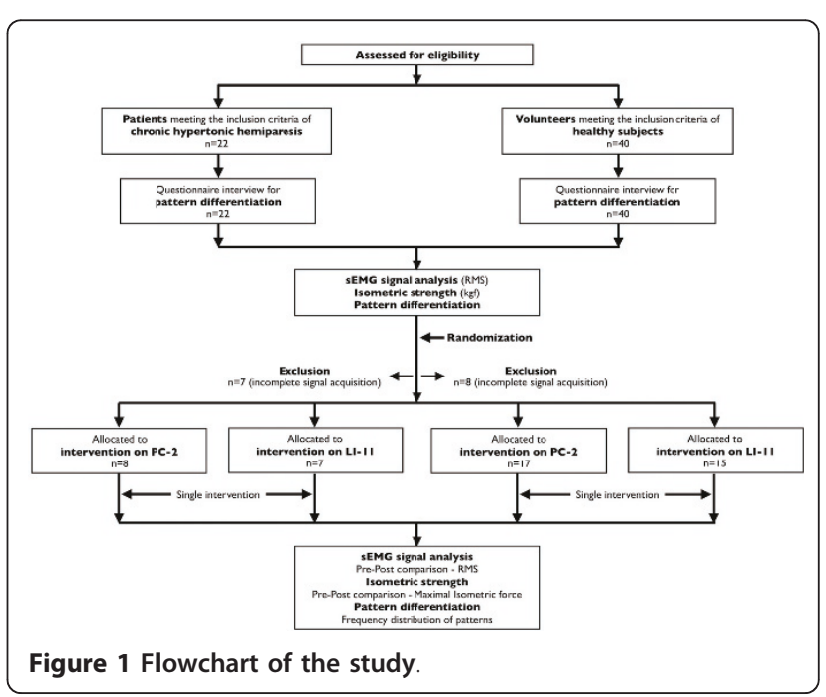




\begin{tabular}{|c|c|c|c|c|c|c|}
\hline & \multicolumn{3}{|c|}{ Healthy subjects } & \multicolumn{3}{|c|}{ Post-stroke patients } \\
\hline & LI11 & PC2 & $P$ & LI11 & PC2 & $P$ \\
\hline Sample size & 15 & 17 & & 7 & 8 & \\
\hline Female & $11(73 \%)$ & $15(88 \%)$ & & $2(29 \%)$ & $5(63 \%)$ & \\
\hline Male & $4(27 \%)$ & $2(12 \%)$ & & $5(71 \%)$ & $3(38 \%)$ & \\
\hline Age (y) & $24.1 \pm 6.0$ & $26.1 \pm 6.6$ & 0.381 & $50.9 \pm 19.2$ & $50.9 \pm 0.3$ & 0.126 \\
\hline Weight (kg) & $66.5 \pm 10.9$ & $63.6 \pm 11.9$ & 0.480 & $74.6 \pm 23.3$ & $70.9 \pm 25.1$ & 0.666 \\
\hline Height (m) & $1.64 \pm 0.07$ & $1.65 \pm 0.06$ & 0.940 & $1.70 \pm 0.33$ & $1.67 \pm 0.32$ & 0.388 \\
\hline Heart rate $(b / \mathrm{min})$ & $74.3 \pm 11.1$ & $75.8 \pm 11.0$ & 0.717 & $76.9 \pm 25.5$ & $81.8 \pm 0.4$ & 0.578 \\
\hline Systolic pressure (mmHg) & $112.0 \pm 16.1$ & $114.7 \pm 15.9$ & 0.637 & $121.4 \pm 37.1$ & $132.5 \pm 0.5$ & 0.286 \\
\hline Diastolic pressure $(\mathrm{mmHg})$ & $65.3 \pm 13.0$ & $67.6 \pm 10.3$ & 0.586 & $80.0 \pm 25.1$ & $80.0 \pm 0.0$ & 1.000 \\
\hline
\end{tabular}

and a chronic (more than six months), partial motor impairment on the upper limb plus the above-cited characteristics of healthy subjects were included. The research included 32 healthy subjects and 15 post-stroke patients, and excluded all cases with incomplete the sEMG signal acquisition or sEMG low-quality signals.

\section{Setup for signal acquisition}

Acupoints were located according to CM $[17,18]$. LI11 was located with the elbow flexed, the radial end of the elbow, at the midpoint of the line connecting Chize (LU5) to the lateral epicondyle of the humerus. PC2 was located on the medial side of the arm, 2 cun below the anterior end of the axillary fold, between long and short heads of biceps brachii muscle. LI11 and PC2 were cleaned with sterile cotton soaped with $70 \%$ alcohol and moistened with a $0.90 \%$ saline solution for the safety of patients and improvement of the sEMG signal conductivity. The sEMG signals were collected using an analog device EMG400C (EMG System, SP, Brazil) connected to a computer by a data acquisition card (NI-6009, 14 bits; National Instruments, Texas, USA). Disposable, auto-adhesive doubledisk electrodes $(\mathrm{Ag} / \mathrm{AgCl}$; diameter $=10 \mathrm{~mm}$; inter-electrode $=10 \mathrm{~mm}$; Hal Industria, SP, Brazil) were placed according to the sEMG for the Non-Invasive Assessment of Muscles (SENIAM) recommendations [19] for the biceps brachii (short head). Active differential electrodes (gain: 20×) were used to collect the sEMG signals from surface electrodes to the analog device. Isometric force signals were acquired by a load cell (range: 0-200 kg; EMG System, SP, Brazil) connected to the same analog device synchronously to the sEMG signals at a sampling rate of $1.0 \mathrm{kHz}$ per channel. Algorithms written in LabVIEW (National Instruments, Texas, USA) were developed to record and process the signals in the time domain.

\section{Procedures and intervention}

All post-stroke patients answered the questions on their clinical status in a questionnaire form (Additional file 1) to differentiate their patterns developed based on [20].
Each volunteer was positioned in a chair with support for the upper limb to remain with the elbow at $90^{\circ}$ (flexion) at the room temperature $\left(21-23^{\circ} \mathrm{C}\right)$. Both acupuncture and signal acquisition were conducted by the same author (APSF) for the consistency of the procedure.

All volunteers executed three repetitions of isometric elbow flexion at MIVC during $5 \mathrm{~s}$ interleaved by $2 \mathrm{~min}$ interval to allow resting and metabolic recovery. In sequence, a sterile, disposable stainless-steel needle $(0.20$ $\times 13 \mathrm{~mm}$, Lizhou, China) was inserted at an angle of $45^{\circ}$ into the selected acupoint towards the direction of $\mathrm{Q} i$ circulation at a depth of approximately $1.5 \mathrm{~cm}$ (the needle length was chosen to minimize the depth of needle insertion among all volunteers). After insertion, the needle was rotated clockwise until the volunteer reported the first Deqi. In manual stimulation, clockwise needle rotation and it was performed immediately after the needle insertion, 5, 10, 15, and $20 \mathrm{~min}$ for $10 \mathrm{~s}$, and accompanied by the report of Deqi. The stimulated traditional functions [21] decreased the RMS and MIVC values. After $20 \mathrm{~min}$, the needle was removed and the signal acquisition was repeated. The surface electrodes were not removed during the acupuncture intervention to avoid the changes due to the electrode position relative to the innervation zone and improve inter-subject reliability.

\section{Reasons for acupoint selection}

Two acupoints were selected for this research on the basis of the previous study [22] on the acupoint prescription for stroke-related disorders. Acupoint Quchi (LI11) was selected as the 'intervention acupoint' among the most cited acupoints. The location and traditional functions of LI11 are related to the motor impairments in post-stroke patterns [17]. Acupoint Tianquan (PC2) was selected as the active 'control acupoint' mainly because of its location.

\section{Signal processing and study outcomes}

The primary outcomes of this study were the RMS and MIVC values estimated from sEMG and load cell 
signals, respectively. Signals were amplified by the analog device (gain: 2,000x) and digitally stored for off-line processing. Load cell signals were lowpass filtered (cutoff frequency: $5 \mathrm{~Hz}$, Butterworth $2^{\text {nd }}$ order, direct and reverse order) and processed by an automatic, doublethreshold method [23] that detected epochs of increased muscular force production for the estimation of MIVC value on each detected epoch. The maximum value was used as the representative value of MIVC at the respective test condition (pre or post intervention). The sEMG signals were bandpass filtered (cut-off frequencies: 5-450 $\mathrm{Hz}$, Butterworth $2^{\text {nd }}$ order, direct and reverse order) and synchronously segmented with the load cell signal. All detected epochs were averaged to represent the RMS value at the respective test condition (pre or post intervention). In addition, the amplitude of a 5-s epoch of baseline signal was estimated with the RMS value for assessment of the lower boundary. The secondary outcome of this study was the frequency distribution of patterns in each group.

\section{Randomization and blinding}

Four parallel groups were randomly generated using a web-based generator. Two sets of numbers (one per group) with 39 numbers per set in range 1-39 were sorted, annotated and inserted into sequentially numbered, opaque sealed envelopes before distribution to all volunteers. Each envelope contained the guidance of the acupuncture intervention: $\mathrm{PC} 2$ in the non-dominant arm of healthy subjects; LI11 in the non-dominant arm of healthy subjects; PC2 in the paretic upper limb; and LI11 in the paretic upper limb. Volunteers received their envelopes in order of admission to the study. The researcher opened the envelope to determine the guidance for acupuncture but the patient did not know the actual acupoint name and function. Additionally, the signals were stored without referring to the selected acupoint to allow a blinded signal processing of the sEMG data.

\section{Statistical analysis}

Sample sizes were estimated from equations suggested to stroke-related outcomes [24]. A sample size of 36 subjects was calculated to observe the reduced RMS values on at least $80 \%$ of healthy subjects after the stimulation at LI11 (intervention acupoint) as compared to a $50 \%$ (random) probability in the same group after the stimulation at PC2 (control acupoint) considering $\alpha=$ $5 \%\left(Z_{\alpha}=1.96\right.$; significance level $)$ and $\beta=80 \%\left(Z_{\beta}=\right.$ 0.84; power of test). This a priori probability was chosen because: 1) needle stimulation on the control acupoint may have effects on RMS values; and 2) variables other than needle stimulation-such as sweating, cooperationcould reduce RMS values by chance in both groups.
The RMS results from the healthy group were used to calculate the sample size for the post-stroke patients, yielding an estimated sample of 14 patients under the same $\alpha$ and $\beta$ values.

Kolmogorov-Smirnov analysis showed that the RMS (average of the three repetitions) and MIVC values (average of the maximal value of each repetition) followed a Gaussian distribution. Intragroup (acupoint) analysis was conducted with unicaudal, paired student's $t$-test to test the null hypothesis that there was no difference in either the RMS or MIVC values between pre and post-intervention. Repeatability was tested with one-way analysis of variance for differences among the three repetitions of isometric contraction separately for acupoint group and test condition (pre and post-intervention). The items in the questionnaire for pattern differentiation were considered as dichotomous variables (present $=1$; absence $=0$ ) used as input variables for the regression equations for pattern differentiation and descriptive statistics of patterns was provided. Chi-square $\left(\chi^{2}\right)$ test was used to test the null hypothesis of no difference in frequency distributions of patterns between healthy and post-stroke subjects. Statistical analysis was performed with SPSS ${ }^{\circledR}$ software version 17 (SPSS Inc., Illinois, USA) and the significance level was considered at $P<0.05$.

\section{Results}

\section{Clinical study 1: Healthy subjects}

The sEMG results and load cell signals were presented in Table 2. The RMS values significantly decreased after the stimulation at both LI11 (pre: $1.392 \pm 0.826 \mathrm{~V}$; post: $0.612 \pm 0.0 .320 \mathrm{~V} ; P=0.002$ ) and PC2 (pre: $1.494 \pm$ $0.826 \mathrm{~V}$; post: $0.623 \pm 0.320 \mathrm{~V} ; P=0.001$ ). This result was not accompanied by a significant decrease in elbow flexion MIVC after the stimulation at LI11 (pre: $22.2 \pm$ $10.7 \mathrm{~kg}$; post: $21.7 \pm 9.5 \mathrm{~kg} ; P=0.288$ ) or $\mathrm{PC} 2$ (pre: 18.8 $\pm 4.6 \mathrm{~kg}$; post: $18.7 \pm 6.0 \mathrm{~kg} ; P=0.468$ ). No significant difference was observed between LI11 and PC2 in the post-pre $(\Delta)$ values of MIVC and RMS $(P=0.340$ and $P$ $=0.391$, respectively).

Repeatability analysis showed no significant difference on the RMS values from three repetitions of maximal voluntary effort before interventions on LI11 $(P=0.885)$ or PC2 $(P=0.892)$, as well as after the stimulation at those acupoints $(P=0.736 ; P=0.906$; respectively $)$. Similarly, the MIVC force was also not significantly different among the three repetitions before the interventions on LI11 $(P=0.864)$ or PC2 $(P=0.977)$, as well as after the stimulation at those acupoints $(P=0.763 ; P=$ 0.986; respectively).

\section{Clinical study 2: Post-stroke patients}

Post-stroke patients exhibited different results (Table 2). Pre-intervention, the MIVC values of all post-stroke 
Table 2 Results from surface electromyography, maximal isometric voluntary contraction and pattern differentiation

\begin{tabular}{|c|c|c|c|c|}
\hline & \multicolumn{2}{|c|}{ Healthy subjects } & \multicolumn{2}{|c|}{ Post-stroke patients } \\
\hline & LI11 & PC2 & LI11 & PC2 \\
\hline \multicolumn{5}{|c|}{ Maximal isometric voluntary force $(\mathrm{kg})$} \\
\hline Pre-intervention & $22.2 \pm 10.7$ & $18.8 \pm 4.6$ & $9.6 \pm 3.9$ & $10.7 \pm 5.6$ \\
\hline Post-intervention & $21.7 \pm 9.5$ & $18.7 \pm 6.0$ & $9.6 \pm 4.7$ & $10.2 \pm 5.3$ \\
\hline$P($ post $\times$ pre $)$ & 0.288 & 0.468 & 0.499 & 0.251 \\
\hline$P(\triangle \mathrm{MIVC}$ LI11 $\times$ PC2) & \multicolumn{2}{|c|}{0.340} & \multicolumn{2}{|c|}{0.303} \\
\hline \multicolumn{5}{|c|}{ Root mean square value $(\mathrm{V})$} \\
\hline Baseline noise & $0.105 \pm 0.010$ & $0.115 \pm 0.026$ & $0.202 \pm 0.085$ & $0.179 \pm 0.052$ \\
\hline Pre-intervention & $1.392 \pm 0.826$ & $1.494 \pm 0.826$ & $0.627 \pm 0.335$ & $0.601 \pm 0.258$ \\
\hline Post-intervention & $0.612 \pm 0.320$ & $0.623 \pm 0.320$ & $0.530 \pm 0.272$ & $0.591 \pm 0.326$ \\
\hline$P($ post $\times$ pre $)$ & 0.002 & 0.001 & 0.187 & 0.398 \\
\hline$P(\triangle \mathrm{RMS} L 111 \times \mathrm{PC} 2)$ & \multicolumn{2}{|c|}{0.391} & \multicolumn{2}{|c|}{0.220} \\
\hline \multicolumn{5}{|l|}{ Identified pattern } \\
\hline Fire heat & $0(0 \%)$ & $3(18 \%)$ & $3(43 \%)$ & $4(50 \%)$ \\
\hline Phlegm dampness & $7(47 \%)$ & $6(35 \%)$ & $2(29 \%)$ & $2(25 \%)$ \\
\hline Qi deficiency & $7(47 \%)$ & $6(35 \%)$ & $1(14 \%)$ & $1(13 \%)$ \\
\hline Yin deficiency & $1(7 \%)$ & $2(12 \%)$ & $1(14 \%)$ & $1(13 \%)$ \\
\hline$P$ (healthy $\times$ patients) & \multicolumn{4}{|c|}{$\chi^{2}=9.759 ; P=0.021$} \\
\hline
\end{tabular}

patients were significantly reduced $(50 \% ; P<0.001)$ compared to healthy subjects. The RMS values were not significantly decreased after the stimulation at both LI11 (pre: $0.627 \pm 0.335 \mathrm{~V}$; post: $0.530 \pm 0.272 \mathrm{~V} ; P=0.187$ ) and PC2 (pre: $0.601 \pm 0.258 \mathrm{~V}$; post: $0.591 \pm 0.326 \mathrm{~V} ; P$ $=0.398)$. This result was not accompanied by a significant decrease on MIVC after the stimulation at LI11 (pre: $9.6 \pm 3.9 \mathrm{~kg}$; post: $9.6 \pm 4.7 \mathrm{~kg} ; P=0.499$ ) or $\mathrm{PC} 2$ (pre: $10.7 \pm 5.6 \mathrm{~kg}$; post: $10.2 \pm 5.3 \mathrm{~kg} ; P=0.251$ ). No significant difference was observed between LI11 and PC2 in the $\triangle$ MIVC and $\triangle$ RMS values $(P=0.303$ and $P$ $=0.220$, respectively).

Repeatability analysis showed no significant difference on the RMS values from three repetitions of maximal voluntary effort before the interventions on LI11 ( $P=$ $0.933)$ or PC2 $(P=0.750)$, as well as after the stimulation at those acupoints $(P=0.998 ; P=0.731$; respectively). Similarly, MIVC force was also not significantly different among the three repetitions before interventions on $\operatorname{LI11}(P=0.480)$ or PC2 $(P=0.970)$, as well as after the stimulation at those acupoints $(P=0.861 ; P=$ 0.881 ; respectively).

\section{Pattern differentiation}

Significant different frequency distributions of patterns were observed among healthy and post-stroke patients $\left(\chi^{2}=9.759 ; P=0.021\right.$; Table 2$)$. Two patterns-"Phlegm dampness" and "Qi deficiency"-were the most commonly identified among healthy subjects (47\% and 35\%, respectively, on each acupoint group LI11 and PC2). Among post-stroke patients, pattern "Fire heat" was the most frequent one (LI11 group: 43\%; PC2 group: 50\%), followed by "Phlegm dampness", "Qi deficiency" and "Yin deficiency" patterns.

\section{Discussion}

This study evaluated the immediate effects of the manual stimulation at acupoints on both myoelectric activity and strength of the biceps brachii in two parallel samples. The results showed that (1) manual acupuncture immediately decreased the RMS but not MIVC values in healthy subjects; (2) manual acupuncture did not immediately decreased RMS or MIVC values in post-stroke patients; (3) LI11 and PC2 elicited similar effects in both healthy and post-stroke groups; and (4) the frequency of identified patterns was different between two groups.

\section{Clinical study 1: Healthy subjects}

The absence in significant difference among the threerepetition test suggested that the average values of RMS and MIVC were representative for further analysis in both parallel groups. The MIVC value of healthy subjects was similar to the previous study $(189 \mathrm{~N} \approx 19.3 \mathrm{~kg})$ [25].

In the group of healthy subjects, the RMS values immediately decreased after the acupuncture, without a respective significant change in MIVC. These results were close to those reported by [13] where similar rationale for acupoint selection (specific indication versus 
location), methods for acupuncture stimulation (rotation every $5 \mathrm{~min}$ ) and sEMG signal-processing details (RMS epoch duration $=5 \mathrm{~s}$ ) were used. However, negative results were observed in studies $[11,12]$ conducted with samples with similar characteristics but with different sEMG signal analysis and acupoint selection. Toma et al. [11] reported the use of an inter-electrode distance of $32 \mathrm{~mm}$ and evaluated the integration of sEMG signal during the entire range of motion divided by contraction time instead of the RMS values. Although the inter-electrode distance may be adequate to record the relative contributions of deep and superficial MU [26], it is larger than currently recommended $(<20 \mathrm{~mm})$ [19]. Tough [12] used similar methods (sample size, intervention duration, etc.), acupoint selection (LI10 instead of LI11) and sEMG analysis (average of three trials, signal sampling frequency, statistical analysis, absolute amplitude values, etc.) except for the epoch duration (10 s each for the RMS estimation) and the lack of assessment of MIVC. Finally, the recommendations from the SENIAM project [19] for electrode positioning and signal processing were not reported [11-13] and may compromise external validity.

\section{Clinical study 2: Post-stroke patients}

In the group of post-stroke patients, neither the RMS nor MIVC values were decreased after acupuncture. Clinical trials on acupuncture for post-stroke MU impairments often tested the efficacy of an intervention by functional outcomes [5-7], while few researches evaluated the physiologic effects of acupuncture intervention in poststroke patients by electromyography. For instance, Zhao et al. [27] evaluated functional and electromyographic parameters (F-wave) and reported significant reduction in spasticity in chronic post-stroke patients submitted to 30 days of acupuncture due to reduced excitability of $\alpha$ motoneurons. Yan and Hui-Chan [28] used functional scales, MIVC and electromyography (co-contraction ratio) in post-stroke patients with acute motor impairments and showed that three weeks of electroacupuncture significantly increased MIVC while co-contraction of medial gastrocnemius and tibialis anterior decreased. These results were attributed to the enhancement of presynaptic inhibition to the hyperactive stretch reflex and disinhibition of voluntary commands to the $\alpha$-motoneurons of the paretic muscles. To the best of our knowledge, this may be the first study to present results on immediate effects of acupuncture stimulation on muscle function in post-stroke patients.

The lack of significant difference in this sample was attributed to a combination of at least four factors. First, RMS pre-intervention values were lower $(42.4 \%)$ in post-stroke patients than healthy subjects probably as a consequence of a lower MIVC (50.0\%)-confirming the negative features of upper limb paresis. This implies in a different "operational point" of the force-MU recruitment relationship and may have influenced the sEMG signal composite pattern. Second, as pre-intervention values were reduced and baseline noise was not different during the 20-min experiment, the possible range for reduction after intervention was compressed and a larger sample might be necessary to detect any possible significant difference, if existent. Third, the neurophysiology of MU recruitment is different for the motor impairments of post-stroke patients [29]. Fourth, neuromuscular activation and muscle unloading were reduced in chronic hemiparetic patients with retained neuromuscular connectivity, which lead to changes in fiber type composition (mainly type-II fibers) in the affected limb [30]. Therefore, the results of this study should be considered as preliminary until a large-sample study confirms the results in this study. Also, it is suggested to perform pre and post-intervention comparisons on smaller percentages of MIVC (e.g. 50\%) to evaluate the effect of the above-cited issues.

\section{A model for SEMG and MIVC interpretation}

Currently, the physiologic explanation for the observed neuromuscular behavior was limited to a "reflex loop" hypothesis and did not have clear explanations on physiologic mechanisms and enrolled structures [11-13]. Based on the current results and consideration on the sEMG signal characteristics and neurophysiology, a theoretical model was proposed for explanation of the results in healthy subjects that was compatible to the adaptive changes in post-stroke subjects and considered acupuncture technique.

Initially, insertion of the acupuncture needle $15 \mathrm{~mm}$ deep into the tissue penetrates the dermis and subcutaneous muscles and creates a small wound (needle radius $=0.2 \mathrm{~mm}$ ) with probable fluid exudation [31]. Rotation of the needle following insertion promotes the mechanical coupling between the needle and connective tissue, and causes winding of tissue surrounding the needle $[32,33]$. This mechanical signal (passive deformation) is transmitted to connective tissue cells and is amplified due to increased tissue displacement [31-33]. The superficial area of tissue deformation may reach $25 \mathrm{~mm}^{2}$ or more around a single needle hole [33] and stimulates several afferent nerve types, evidenced by the variety of subjective report of the Deqi sensation [34] most frequently described as "aching" or "soreness" [35]. CM theory states that Deqi must be achieved and sustained throughout the session to maximize therapeutic effects and thus needle is intermittently manipulated at shorttime intervals ( 3 to $5 \mathrm{~min}$ ). Finally, a significant higher pullout force is necessary for needle extraction from real acupoints [36] with additional tissue injury due to 
connective tissue adherence to the needle tip. Altogether, $\mathrm{CM}$ acupuncture intervention (= needle insertion + intermittent manipulation with Deqi + needle extraction) evokes an uncomfortable sensation during the entire session and sometimes is prolonged after intervention.

Regarding the sEMG signal interpretation, it was reported that decreased MU discharge rate with pain was accompanied by changes in the population of $\mathrm{MU}$ used to maintain force [37]. Moreover, pain decreased MU synchronization with a consequent decrease in the sEMG amplitude estimators, mainly due to amplitude cancellation between positive and negative phase of the MU action potential [38]. Hence, the decreased RMS (healthy group: $-56 \%$ to $-58 \%$; post-stroke patients: $-2 \%$ to $-16 \%$ ) and sustained MIVC (healthy group: $0 \%$ to $-2 \%$; post-stroke patients: $0 \%$ to $-5 \%$ ) values observed after the stimulation at both LI11 and PC2 were fully consistent with the proposed model that the sustained painful sensation may decreased MU discharge but does not significantly changed MIVC. The lack of acupoint specific effects was also reported by other studies with sEMG and clinical outcomes $[11,12,39]$ and was consistent with the results of the present study and the proposed model. Further studies are necessary to validate the proposed model, especially if the long-term effects were attributed to multiple short-term acupuncture stimulation on the sEMG variables.

\section{Methodology}

As an important muscle commonly affected in upper neuron lesions, the biceps brachii is well suited for sEMG analysis because of its long, parallel fibers with a main innervation zone often located at the muscle belly. Although our parallel groups exhibit different characteristics on gender and age at baseline, a recent study found no significant effect of gender and age (range: $<10$ to 70 years old) subjects regarding electromyographic parameters obtained from the biceps brachii [40] and thus we believe that this factor had no major influence on the obtained results. The RMS value fails to yield a general relationship with muscle strength [40]. Hence, changes in the RMS values of sEMG after an intervention may not rigorously reflect any altered level of neural drive to the muscle [41]. Additionally, a recent study [42] have demonstrated that the high frequency band $(>440 \mathrm{~Hz})$ of sEMG signals allows an accurate estimation of the forceRMS relationship and needs further attention to assess the robustness of the proposed model to changes in the sEMG processing techniques.

\section{Pattern differentiation}

In the present study, CM pattern differentiation was performed by an automated model designed for post- stroke patients from a large sample study [20]. The interesting result was that despite the small sample size of post-stroke patients, this study supported the CM theory that different frequency distributions of patterns may occurs between them and healthy subjects. However, a larger study sample and a proper study design may be necessary to provide more definitive conclusions. Nevertheless, the obtained results may be used to design studies on pattern differentiation, e.g. determining sample sizes based on prevalence of patterns. As a limitation, the binary logistic regression method was not validated and its diagnostic accuracy is unknown. Moreover, it was not yet applied to a sample of healthy subjects until now. Ongoing research on this topic included the determination of diagnostic accuracy of the method in post-stroke patients, the establishment of cutoff points for probabilities estimates from each regression equation, and comparison with other automated methods for pattern differentiation [43-45]. As it was argued that pattern differentiation may lead to best therapeutic results [46], the automated model with highest diagnostic performance should be used in future clinical trials to determine the therapeutic intervention in a reproducible manner.

\section{Conclusion}

Manual acupuncture provides sufficient neuromuscular stimuli to promote immediate changes in MU gross recruitment without repercussion in maximal force output in healthy subjects. Post-stroke patients did not exhibit significant reduction on myoelectric activity and maximal force output after manual acupuncture and needs further evaluation with a larger sample.

\section{Additional material}

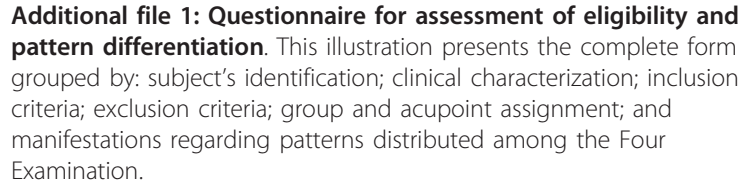

Additional file 1: Questionnaire for assessment of eligibility and pattern differentiation. This illustration presents the complete form grouped by: subject's identification; clinical characterization; inclusion criteria; exclusion criteria; group and acupoint assignment; and manifestations regarding patterns distributed among the Four Examination.

\section{Abbreviations}

CM: Chinese medicine; sEMG: Surface electromyography; RMS: Root meansquared value; MU: Motor unit; MIVC: Maximal isometric voluntary contraction; $\triangle \mathrm{RMS}$ : Difference between post and pre RMS values; $\triangle \mathrm{MIVC}$ : Difference between post and pre MIVC values; SENIAM: Surface electromyography for the non-invasive assessment of muscles.

\section{Acknowledgements}

We are in debit to Professor Marcos Soares who is the coordinator of the Nucleus of Study and Research of the Fluminense Association of Rehabilitation (AFR). We also would like to thank Dr. Valéria Marques (assistant of the Nucleus of Study and Research of the Fluminense Association of Rehabilitation, AFR) for her great contribution in patient 
recruitment. Also, the authors would like to acknowledge the helpful comments from the reviewers.

\section{Author details}

'Laboratory of Human Motion Analysis, Post-graduation Program of Rehabilitation Science, Centro Universitário Augusto Motta, Rio de Janeiro, Brazil. ${ }^{2}$ Graduation Program of Physical Therapy, Universidade Salgado de Oliveira, Niterói, Brazil.

\section{Authors' contributions}

APSF designed the study, conducted the questionnaire interview, selected the subjects, and wrote the manuscript. ASF designed the study, developed the computational methods for pattern differentiation, performed the statistical analysis, and wrote the manuscript. All authors read and approved the final version of the manuscript.

\section{Competing interests}

The authors declare that they have no competing interests.

Received: 18 November 2011 Accepted: 14 March 2012

Published: 14 March 2012

\section{References}

1. World Health Organization: The Atlas of Heart Disease and Stroke Geneva: WHO; 2004

2. World Health Organization: Cardiovascular diseases (CVDs). Fact sheet $N^{\circ} 317$ 2009.

3. Goldstein LB, Adams R, Alberts MJ, Appel LJ, Brass LM, Bushnell CD, Culebras A, DeGraba TJ, Gorelick PB, Guyton JR, Hart RG, Howard G, KellyHayes M, Nixon JVI, Sacco RL: Primary Prevention of Ischemic Stroke. Stroke 2006, 37:1583-1633.

4. Rundek T, Sacco RL: Risk Factor Management to Prevent First Stroke. Neurol Clin 2008, 26:1007-ix.

5. Wu HM, Tang JL, Lin XP, Lau JTF, Leung PC, Woo J, Li Y: Acupuncture for stroke rehabilitation. Cochrane Database of Systematic Reviews 2006, 3: CD004131.

6. Park J, Hopwood V, White AR, Ernst E: Effectiveness of acupuncture for stroke: A systematic review. J Neurol 2001, 248:558-563.

7. Sze FKH, Wong E, Or KKH, Lau J, Woo J: Does Acupuncture Improve Motor Recovery After Stroke? A Meta-Analysis of Randomized Controlled Trials. Stroke 2002, 33:2604-2619.

8. Fragoso APS, Ferreira AS: A fisiopatologia do acidente vascular cerebral segundo a medicina Chinesa. Revista Augustus (Rio de Janeiro. Impresso);.

9. Fragoso APS: Ensaios clínicos com acupuntura: questões bioéticas e Metodológicas. Perspectivas online 2010, 4:151-158[http://www.sumarios. org/sites/default/files/pdfs/53013 6204.PDF].

10. Ferreira AS, Guimarães FS, Silva JG: Aspectos metodológicos da eletromiografia de superfície: Considerações sobre os sinais e processamentos para estudo da função neuromuscular. Rev Bras Cienc Esporte 2010, 31:11-30[http://www.rbceonline.org.br/revista/index.php/RBCE/ article/view/300/406]

11. Toma K, Conatser RR Jr, Gilders RM, Hagerman FC: The effects of acupuncture needle stimulation on skeletal muscle activity and performance. J Strength Cond Res 1998, 12:253-257.

12. Tough E: Lack of effect of acupuncture on electromyographic activity: a randomised controlled trial in healthy volunteers. Acupunct Med 2006, 24:55-60.

13. Costa LA, Araújo JE: The immediate effects of local and adjacent acupuncture on the tibialis anterior muscle: a human study. Chin Med 2008, 3:17.

14. Fragoso APS, Ferreira AS: Evaluation of the immediate effects of manual acupuncture on brachial bicep muscle function in healthy individuals and poststroke patients: a study protocol of a parallel-group randomized clinical trial. J Chin Integr Med 2012, 10:303-309.

15. Moher D, Hopewell S, Schulz KF, Montori V, Gøtzsche PC, Devereaux PJ, Elbourne D, Egger M, Altman DG: Consolidated Standards of Reporting Trials Group. CONSORT 2010 Explanation and Elaboration: Updated guidelines for reporting parallel group randomised trials. J Clin Epidemiol 2010, 63:e1-37.

16. MacPherson H, Altman DG, Hammerschlag R, Youping L, Taixiang W, White A, Moher A, on behalf of the STRICTA Revision Group: Revised
STandards for Reporting Interventions in Clinical Trials of Acupuncture (STRICTA): Extending the CONSORT Statement. J Altern Complement Med 2010, 16:ST1-ST14.

17. Maciocia G: The foundations of Chinese medicine: a comprehensive text for acupuncturists and herbalists Churchill Livingstone; 1989.

18. World Health Organization: WHO standard acupuncture point locations in the Western Pacific Region Geneva: WHO; 2008

19. Hermens HJ, Freriks B, Merletti R, Stegeman D, Blok J, Rau G, DisselhorstKlug C, Hägg G: European Recommendations for Surface Electromyography. Results of the SENIAM project, 2 1999, ISBN: 90-75452-15-2.

20. Kim HJ, Bae HS, Park SU, Moon SK, Park JM, Jung WS: Clinical Approach to the Standardization of Oriental Medical Diagnostic Pattern Identification in Stroke Patients. Evidence-Based Complement Alternat Med 2011, 201(1):7.

21. Moncayo R, Moncayo $\mathrm{H}$ : Evaluation of Applied Kinesiology meridian techniques by means of surface electromyography (sEMG): demonstration of the regulatory influence of antique acupuncture points. Chin Med 2009, 4:9

22. Fragoso APS, Ferreira AS: Statistical distribution of acupoint prescriptions for sensory-motor impairments in post-stroke subjects. Chin J Integr Med 2012.

23. Reaz MBI, Hussain MS, Mohd-Yasin F: Techniques of EMG signal analysis: detection, processing, classification and applications. Biol Proced Online 2006, 8:11-35.

24. The Optimising Analysis of Stroke Trials (OAST) collaboration: Calculation of sample size for stroke trials assessing functional outcome: comparison of binary and ordinal approaches. Int J Stroke 2008, 3:78-84.

25. Nygaard $E$, Houston M, Suzuki $Y$, Jørgensen $K$, Saltin B: Morphology of the brachial biceps muscle and elbow flexion in man. Acta Physio/ Scand 1983, 117:287-292.

26. Hermens HJ, Freriks B, Disselhorst-Klug C, Rau G: Development of recommendations for SEMG sensors and sensor placement procedures. $J$ Electromyogr Kinesiol 2000, 10:361-374.

27. Zhao JG, Cao CH, Liu CZ, Han BJ, Zhang J, Li ZG, Yu T, Wang XH, Zhao H, $\mathrm{Xu} \mathrm{ZH}$ : Effect of acupuncture treatment on spastic states of stroke patients. J Neurolog Sci 2009, 276:143-147.

28. Yan T, Hui-Chan CWY: Transcutaneous electrical stimulation on acupuncture points improved muscle function in subjects after acute stroke: a randomized controlled trial. J Rehabil Med 2009, 41:312-316.

29. Shepherd RB: Exercise and Training to Optimize Functional Motor Performance in Stroke: Driving Neural Reorganization? Neural Plast 2001 8:121-129.

30. Hafer-Macko CE, Ryan AS, Ivey FM, Macko R: Skeletal muscle changes after hemiparetic stroke and potential beneficial effects of exercise intervention strategies. J Rehab Res Develop 2008, 45:261-272.

31. Langevin HM, Konofagou EE, Badger GJ, Churchill DL, Fox JR, Ophir J, Garra BS: Tissue displacements during acupuncture using ultrasound elastography techniques. Ultrasound Med Biol 2004, 30:1173-1183.

32. Langevin HM, Churchill DL, Cipolla MJ: Mechanical signaling through connective tissue: a mechanism for the therapeutic effect of acupuncture. FASEB J 2001, 15:2275-2001.

33. Langevin HM, Churchill DL, Wu J, Badger GJ, Yandow JA, Foxá JR, Krag MH: Evidence of Connective Tissue Involvement in Acupuncture. FASEB J 2002, 16:872-874

34. Roth LU, Maret-Maric A, Adler RH, Neuenschwander BE: Acupuncture Points have Subjective (Needling Sensation) and Objective (Serum Cortisol Increase) Specificity. Acupunct Med 1997, 15:2-5.

35. Hui KK, Nixon EE, Vangel MG, Liu J, Marina O, Napadow V, Hodge SM, Rosen BR, Makris N, Kennedy DN: Characterization of the "deqi" response in acupuncture. BMC Complement Altern Med 2007, 7:33.

36. Langevin HM, Churchill DL, Fox JR, Badger GJ, Garra BS, Krag MH: Biomechanical response to acupuncture needling in humans. J Appl Physiol 2001, 91:2471-2478.

37. Tucker K, Butler J, Graven-Nielsen T, Riek S, Hodges P: Motor unit recruitment strategies are altered during deep-tissue pain. J Neurosci 2009, 29:10820-10826.

38. Keenan KG, Farina D, Maluf KS, Merletti R, Enoka RM: Influence of amplitude cancellation on the simulated surface electromyogram. J Appl Physiol 2005, 98:120-131.

39. Zhang H, Bian Z, Lin Z: Are acupoints specific for diseases? A systematic review of the randomized controlled trials with sham acupuncture controls. Chin Med 2010, 5:1. 
40. Kaplani PA, Pattichis CS, Hadjileontiadis LJ, Roberts VC: Surface EMG analysis on normal subjects based on isometric voluntary contraction. J Electromyogr Kinesiol 2009, 19:157-171.

41. Farina D, Merletti R, Enoka RM: The extraction of neural strategies from the surface EMG. J Appl Physiol 2004, 96:1486-1495.

42. Potvin JR, Brown SH: Less is more: high pass filtering, to remove up to 99\% of the surface EMG signal power, improves EMG-based biceps brachii muscle force estimates. J Electromyogr Kinesiol 2004, 14:389-399.

43. de Sá Ferreira A: Statistical validation of strategies for Zang-fu single pattern differentiation. J Chin Integr Med 2008, 6:1109-1116.

44. Ferreira AS: Diagnostic accuracy of pattern differentiation algorithm based on traditional Chinese medicine theory: a stochastic simulation study. Chin Med 2009, 4:24.

45. Sá Ferreira A: Misdiagnosis and undiagnosis due to pattern similarity in Chinese medicine: a stochastic simulation study using pattern differentiation algorithm. Chin Med 2011, 6:13.

46. Ferreira AS, Lopes AJ: Chinese Medicine Pattern Differentiation and Its Implications for Clinical Practice. Chin J Integr Med 2011, 17:818-823.

doi:10.1186/1749-8546-7-7

Cite this article as: Fragoso and Ferreira: Immediate effects of acupuncture on biceps brachii muscle function in healthy and poststroke subjects. Chinese Medicine 2012 7:7.

\section{Submit your next manuscript to BioMed Central and take full advantage of:}

- Convenient online submission

- Thorough peer review

- No space constraints or color figure charges

- Immediate publication on acceptance

- Inclusion in PubMed, CAS, Scopus and Google Scholar

- Research which is freely available for redistribution

Submit your manuscript at www.biomedcentral.com/submit 